\title{
Refinements of the Chern-Dold character: cocycle additions in differential cohomology
}

\author{
Markus Upmeier ${ }^{1}$
}

Received: 13 April 2014 / Accepted: 6 March 2015 / Published online: 24 April 2015

(C) Tbilisi Centre for Mathematical Sciences 2015

\begin{abstract}
The Chern-Dold character of a cohomology theory $E$ is a canonical transformation $E \rightarrow H V$ to ordinary cohomology. A spectrum representing $E$ gives homotopy theoretic cocycles for $E$, while $H V$ can be represented by singular cocycles. We construct a refinement of the Chern-Dold character to a transformation of the cocycle categories that takes the homotopical composition to the addition of singular cocycles. This is applied to construct additive structures at the level of differential cocycles for generalized differential cohomology.
\end{abstract}

Keywords Chern character - Chern-Dold character - Generalized cohomology · Differential cohomology

Mathematics Subject Classification 55N20

\section{Introduction}

The Chern-Dold character (see [2]) is a natural transformation from an arbitrary generalized cohomology theory $E$ to ordinary cohomology with coefficients in the graded coefficient vector space $V^{*}=E^{*}\left(S^{0}\right) \otimes_{\mathbb{Z}} \mathbb{R}$ :

Communicated by Ronald Brown.

This work benefitted from an ARC of the Communauté française de Belgique and from an Inter-university Attraction Pole from the Politique Scientifique de Belgique.

$凶 \quad$ Markus Upmeier

markus.upmeier@math.uni-augsburg.de

1 Universität Augsburg, Universitätsstrasse 14, 86159 Augsburg, Germany 


$$
\operatorname{ch}: E^{*}(X) \longrightarrow H^{*}(X ; V)=\prod_{i+j=*} H^{i}\left(X ; V^{j}\right) \quad\left(X \in \mathcal{T} \text { op }^{*}\right)
$$

(cohomology, cochain groups, etc. for pointed spaces are always reduced). Hence for $E=H \mathbb{Z}$ we get the standard map $H^{*}(X, \mathbb{Z}) \rightarrow H^{*}(X, \mathbb{R})$. For topological $K$-theory, we get the ordinary Chern character $K^{0 / 1}(X) \rightarrow H^{\text {ev/od }}(X)$. Note that the graded coefficient vector space $V$ depends on the fixed choice of generalized cohomology theory $E$.

Ordinary cohomology can be represented by the abelian group of singular cocycles $Z^{n}(X ; V)$. These form the objects of a strict monoidal category of cocycles $\mathscr{Z}^{n}(X)$. Similarly, given a spectrum $\left(E_{n}, \varepsilon_{n}\right)$ representing $E$ (with homeomorphisms $\varepsilon_{n}^{\text {adj }}: E_{n} \rightarrow \Omega E_{n+1}$, which may always be arranged), maps $X \rightarrow E_{n}$ give cocycles for generalized cohomology. Loop composition in either direction gives two binary operations, identifying $E_{n}$ with $\Omega^{2} E_{n+2}$. Endowed with these, the cocycles $\mathcal{M a p}\left(X, E_{n}\right)$ for generalized cohomology form a 2-monoidal category (see Sect. 2), a more sophisticated algebraic object than an abelian group.

We shall construct a refined Chern-Dold character between the cocycle categories in such a way that it preserves the algebraic structure (strict addition of singular cocycles, loop composition). Here, the category of singular cocycles $\mathscr{Z}^{n}(X)$ is viewed as a 2monoidal category in which both monoidal structures, given by addition of cocycles, coincide (see Definition 7).

Theorem 1 For any generalized cohomology theory $E$ and representing spectrum $\left(E_{n}, \varepsilon_{n}\right)$, there exists a natural family of 2-monoidal functors

$$
\operatorname{ch}_{X}: \mathcal{M a p}\left(X, E_{n}\right) \rightarrow \mathscr{Z}^{n}(X) .
$$

On isomorphism classes of objects, the functors (2) reduce to (1).

The notation is established in Sect. 2 where we also review the theory of 2-monoidal categories. Theorem 1 is proven in Sect. 4.2 after having explained in Sect. 3 that the construction of (2) passes through an intermediate step which mediates between the algebraic and homotopical point of view.

One motivation is the following application (Sect. 5): Let $E_{n}$ be a fixed choice of $\Omega$-spectrum representing the generalized cohomology theory $E$. Recall from [4, Definitions $4.34,4.1]$ that a differential $n$-cocycle on a manifold $M$ (with respect to $E$ ) consists of a continuous map $c: M \rightarrow E_{n}$, a differential form $\omega \in \Omega^{n}(M ; V)$, and a cochain $h \in C^{n-1}(M ; V)$ satisfying

$$
\delta h=\omega-c^{*} \iota_{n} .
$$

(here, $\iota_{n} \in Z^{n}\left(E_{n} ; V\right)$ denote fundamental cocycles, see Sect. 4.1. A differential cocycle on $M \times[0,1]$ is regarded as an equivalence between the two cocycles on the boundary. The Hopkins-Singer differential cohomology group $\hat{E}^{n}(M)$ is by definition the set of equivalences classes of differential $n$-cocycles on $M$.

Differential cocycles can also be organized into a category $\hat{\mathcal{E}}^{n}(M)$. We have the forgetful functor $2 \mathcal{M}$ on $\rightarrow \mathcal{C}$ at along which we shall lift the functor $\hat{\mathcal{E}}$. In other 
words, we will construct natural 2-monoidal structures on the categories of differential cocycles. This is essentially a consequence of Theorem 1. Our main application is the following (nearly equivalent) statement:

Theorem 2 For every choice of fundamental cocycles there exist reduced cochains $A_{n} \in C^{n-1}\left(E_{n} \times E_{n} ; V\right)$ satisfying coherence relations (see Sect. 5) so that

$$
\left(c_{1}, \omega_{1}, h_{1}\right)+\left(c_{2}, \omega_{2}, h_{2}\right)=\left(\alpha_{n}\left(c_{1}, c_{2}\right), \omega_{1}+\omega_{2}, h_{1}+h_{2}+\left(c_{1}, c_{2}\right)^{*} A_{n}\right)
$$

gives an abelian group structure on the Hopkins-Singer differential extension $\hat{E}$.

In many cases it is important to have control of the algebraic structure at the level of differential cocycles. This is in sharp contrast to [4], where it is proven that the cohomology groups $\hat{E}^{n}(M)$ possess some abstract abelian group structure (they are identified as the homotopy groups of a spectrum whose structure maps are only abstractly chosen by a cofibrant replacement in a diagram model category, i.e., a choice of functorial sections): there is then no way of deciding which differential cocycle represents the sum.

\section{Theory of 2-monoidal categories}

The following is a special case of $[6$, Section 5] (or [1, 6.1]) where the units coincide:

Definition 3 A 2- $(\mathcal{C}, \oplus, \ominus, I, \zeta)$ is a category $\mathcal{C}$ having two monoidal structures $\oplus, \ominus$ sharing a unit $I$ and a natural 'interchange' isomorphism

$$
\zeta_{A, B, C, D}:(A \ominus B) \oplus(C \ominus D) \rightarrow(A \oplus C) \ominus(B \oplus D)
$$

We require $I \oplus I=I=I \ominus I$ which along with (4) shall endow both

$$
\text { (1) }(\mathcal{C} \times \mathcal{C}, \ominus \times \ominus) \rightarrow(\mathcal{C}, \ominus), \quad \ominus:(\mathcal{C} \times \mathcal{C}, \oplus \times \oplus) \rightarrow(\mathcal{C}, \oplus)
$$

with the structure of monoidal functors.

A 2-monoidal functor $F: \mathcal{C} \rightarrow \mathcal{D}$ has monoidal structures $F^{\oplus}:(\mathcal{C}, \oplus) \rightarrow(\mathcal{D}, \oplus)$, $F^{\ominus}:(\mathcal{C}, \ominus) \rightarrow(\mathcal{D}, \ominus)$ whose unit constraints $F^{\oplus, \ominus}(I)=I$ are the identity. We require commutative diagrams for all objects $A, B, C, D$

$$
\begin{aligned}
& F((A \ominus B) \oplus(C \ominus D)) \stackrel{F\left(\zeta^{\mathcal{C}}\right)}{\longrightarrow} F((A \oplus C) \ominus(B \oplus D)) \\
& F_{A \ominus B, C \ominus D}^{\oplus} \downarrow \downarrow F_{A \oplus C, B \oplus D}^{\ominus} \\
& F(A \ominus B) \oplus F(C \ominus D) \quad F(A \oplus C) \ominus F(B \oplus D) \\
& F_{A, B}^{\ominus} \oplus F_{C, D}^{\ominus} \downarrow \mid \downarrow F_{A, C}^{\oplus} \ominus F_{B, D}^{\oplus} \\
& (F A \ominus F B) \oplus(F C \ominus F D) \underset{\zeta^{\mathcal{D}}}{\longrightarrow}(F A \oplus F C) \ominus(F B \oplus F D) \text {. }
\end{aligned}
$$


Restricting to small categories $\mathcal{C}$, these definitions give a category $2 \mathcal{M}$ on. We call $F$ an equivalence if it is an equivalence of the underlying categories.

We assume familiarity with monoidal categories as presented in [8]. Equality of 2monoidal functors $F=G$ means that both monoidal structures $F^{\oplus}=G^{\oplus}, F^{\ominus}=G^{\ominus}$ agree. $F$ is called strict if both $F^{\oplus}, F^{\ominus}$ are strict. Restricting to such functors gives the subcategory $2 \mathcal{M o n}_{\text {strict }}$. Denote by $\mathcal{C}$ at $(\mathcal{M}$ onC $\mathcal{C}$ at) the category of small (monoidal) categories.

We think of a functor $\mathcal{C}: \mathcal{I} \rightarrow \mathcal{C}$ at as a (natural) family of categories (by $\mathcal{C}$ at we mean the large category of all small categories and similarly for $2 \mathcal{M}$ on). By a 2-monoidal structure on $\mathcal{C}$ we mean a lift to a functor $\hat{\mathcal{C}}: \mathcal{I} \rightarrow 2 \mathcal{M}$ on along the forgetful functor $2 \mathcal{M}$ on $\rightarrow \mathcal{C}$ at: for every $X \in \mathcal{I}$ we have 2-monoidal categories $\mathcal{C}_{X}$ and every morphism $f \in \mathcal{I}(X, Y)$ gives a 2-monoidal functor $\mathcal{C}(f): \mathcal{C}_{X} \rightarrow \mathcal{C}_{Y}$. A natural transformation $F: \mathcal{C} \Rightarrow \mathcal{D}$ between two families $\mathcal{C}, \mathcal{D}: \mathcal{I} \rightarrow 2 \mathcal{M}$ on may be viewed as a (natural) family of 2-monoidal functors: for every $X \in \mathcal{I}$ we have a 2-monoidal functor $F_{X}: \mathcal{C}_{X} \rightarrow \mathcal{D}_{X}$. Naturality means that for $f: X \rightarrow Y$ in $\mathcal{I}$ we get a commutative diagram in $2 \mathcal{M}$ on:

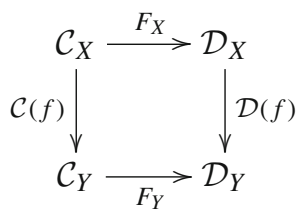

The motivating example for the theory of 2-monoidal categories is the following:

Example 4 The fundamental groupoid $\Pi_{1} \Omega^{2} X$ of the double loop space of a space $X \in \mathcal{T}$ op* $^{*}$ has monoidal structures by vertical and horizontal composition:

$$
\begin{aligned}
& f \oplus g(s, t)= \begin{cases}f(2 s, t), & s \leq 1 / 2, \\
g(2 s-1, t), & s \geq 1 / 2,\end{cases} \\
& f \ominus g(s, t)= \begin{cases}f(s, 2 t), & t \leq 1 / 2, \\
g(s, 2 t-1), & t \geq 1 / 2 .\end{cases}
\end{aligned}
$$

Here we view a double loop as a map $f, g:[0,1]^{2} \rightarrow X$. The associativity and unit constraints are given by the standard homotopies that are used to show that the fundamental group is indeed a group - for formulas see [9, 4.1.1]. The interchange $\zeta$ is the identity and the common unit $I$ is the base-point map. Since maps $X \rightarrow Y$ preserve $\left(1, \ominus, I\right.$ we get a functor $\Pi_{1} \Omega^{2}: \mathcal{T}$ op $^{*} \rightarrow 2 \mathcal{M}$ on $_{\text {strict }}$.

There is a unique way to transport a 2-monoidal structure along an isomorphism $F: \mathcal{C} \rightarrow \mathcal{D}$ of categories, turning $F$ into a strict 2-monoidal functor [so $X \oplus Y=$ $F^{-1}(F X \oplus F Y)$ and $F \zeta^{\mathcal{C}}=\zeta^{\mathcal{D}}$ ]. As usual, uniqueness implies functoriality: any functor $\mathcal{C}: \mathcal{I} \rightarrow \mathcal{C}$ at naturally isomorphic to $\mathcal{D}: \mathcal{I} \rightarrow 2 \mathcal{M}$ on may be uniquely lifted along the forgetful functor to $\hat{\mathcal{C}}: \mathcal{I} \rightarrow 2 \mathcal{M}$ on, making every component $\hat{\mathcal{C}}_{X} \rightarrow \mathcal{D}_{X}$ of the transformation a strict 2-monoidal functor. 
Definition 5 For $X \in \mathcal{T}$ op*, let $\mathcal{M a p}\left(X, E_{n}\right)=\Pi_{1} E_{n}^{X}$ be the fundamental groupoid of the pointed mapping space (pointed maps $X \rightarrow E_{n}$ and homotopies). The structure maps induce natural isomorphisms $\mathcal{M a p}\left(X, E_{n}\right) \cong \Pi_{1} \Omega^{2} E_{n+2}^{X}$ to 2-monoidal categories from Example 4. Transporting, we get

$$
\mathcal{M a p}\left(-, E_{n}\right): \mathcal{T} \text { op }^{*} \rightarrow 2 \mathcal{M} \text { on }
$$

(Recall that $\left(E_{n}, \varepsilon_{n}\right)$ is a spectrum representing the cohomology theory $E$ ).

Examples 6 (i) Let $A$ be a topological or simplicial abelian group. On $\Pi_{1} A$ we take $(1)=\ominus=+, I=0$, and the identity as the interchange. Since + and 0 are preserved by group homomorphisms, we get a functor $\Pi_{1}: s \mathcal{A b} \rightarrow 2 \mathcal{M}_{\text {on }}$ strict .

(ii) Any monoidal category $(\mathcal{C}, \otimes)$ may be regarded as being 2-monoidal by taking $(1)=\ominus=\otimes$ and $\zeta=\mathrm{id}$. This yields a functor $\mathcal{M}$ onC at $\rightarrow 2 \mathcal{M}$ on.

(iii) For every cochain complex $\left(C^{*}, d\right)$ and $n \in \mathbb{Z}$, define a strict monoidal category $\mathscr{Z}^{n}$ of $n$-cocycles: objects are $x \in C^{n}$ with $d x=0$. A morphism $x \rightarrow y$ consists of an $\operatorname{im}(d)$-coset of elements $u \in C^{n-1}$ with $d u=x-y$. Composition and the monoidal structure are both given by addition. Combined with (ii) we obtain for each $n \in \mathbb{Z}$ a functor $\mathscr{Z}^{n}: \mathcal{C h} \rightarrow 2 \mathcal{M}_{\text {strict }}$ on cochain complexes.

Recall $C^{*}(X ; V)=\prod_{i+j=*} C^{i}\left(X ; V^{j}\right)$ for a graded vector space $V^{*}$. Being fixed in our discussion as $V=E^{*}\left(S^{0}\right) \otimes_{\mathbb{Z}} \mathbb{R}$, we will simply write $C^{*}(X)=C^{*}(X ; V)$. Similarly, we shall write $Z^{*}(X)=Z^{*}(X ; V)$ for the singular cocycles of $X$ with coefficients in $V$. For chains groups $C_{*}(X)$, this convention is not adopted.

Definition 7 The reduced cochain complex $C^{*}(-; V)$ from $\mathcal{T}$ op* to $\mathcal{C}$ h composed with (iii) gives the functor $\mathscr{Z}^{n}: \mathcal{T}_{\mathrm{op}^{*}} \rightarrow 2 \mathcal{M} \mathrm{on}_{\text {strict }}$.

The proofs of the following two propositions are given in "Appendix".

Proposition 8 Let $\mathcal{C}, \mathcal{D}, \mathcal{E}$ be 2-monoidal categories and suppose $F: \mathcal{C} \rightarrow \mathcal{D}$, $G: \mathcal{D} \rightarrow \mathcal{E}$ are (ordinary) functors of the underlying categories. Let $H=G \circ F$.

1. If $F$ is an equivalence and $F, H$ have 2-monoidal structures, then $G$ has a unique 2-monoidal structure such that $H=G \circ F$ as 2-monoidal functors.

2. If $G$ is an equivalence and $G, H$ have 2-monoidal structures, then $F$ has a unique 2-monoidal structure such that $H=G \circ F$ as 2-monoidal functors.

Proposition 9 Let $\mathcal{C}: \mathcal{I} \rightarrow \mathcal{C}$ at, $\mathcal{D}: \mathcal{I} \rightarrow 2 \mathcal{M}$ on be functors. Suppose $F: \mathcal{C} \Rightarrow \mathcal{D}$ is a nat. transformation of $\mathcal{C}$ at-valued functors whose components are equivalences

$$
F_{X}: \mathcal{C}_{X} \stackrel{\sim}{\rightarrow} \mathcal{D}_{X}, \quad X \in \mathcal{I}
$$

Then we may lift $\mathcal{C}$ to $\hat{\mathcal{C}}: \mathcal{I} \rightarrow 2 \mathcal{M}$ on and promote $F$ to a natural family of 2-monoidal equivalences $\hat{F}_{X}: \hat{\mathcal{C}}_{X} \rightarrow \mathcal{D}_{X}$ (i.e., a natural transformation $\left.F: \hat{\mathcal{C}} \Rightarrow \mathcal{D}\right)$.

Of course, this also holds in the dual situation $\mathcal{C}: \mathcal{I} \rightarrow 2 \mathcal{M}$ on, $\mathcal{D}: \mathcal{I} \rightarrow \mathcal{C}$ at. We emphasize that the lift $\hat{\mathcal{C}}$ is not unique, but can still be chosen functorially. 
Example 10 On the category $\mathcal{K}$ an* of pointed Kan complexes, consider

$$
\mathcal{C}: \mathcal{K} \mathrm{an}^{*} \stackrel{\Pi_{1} \Omega^{2}}{\longrightarrow} \mathcal{C} \text { at, } \mathcal{D}: \mathcal{K} \mathrm{an}^{*} \stackrel{|\cdot|}{\longrightarrow} \mathcal{T} \mathrm{op}^{*} \stackrel{\Pi_{1} \Omega^{2}}{\longrightarrow} 2 \mathcal{M} \text { on }
$$

( $F_{X}$ is induced by geometric realization of points and paths in $X$ ). Hence the fundamental groupoids $\Pi_{1} \Omega^{2} K$ for pointed Kan complexes (see [3]) can be given functorial 2-monoidal structures $\Pi_{1} \Omega^{2}: \mathcal{K}$ an $^{*} \rightarrow 2 \mathcal{M}$ on.

One of the main results of [6, Section 5] (or see [1, Proposition 6.11]) is that there is an equivalence from $2 \mathcal{M}$ on to braided monoidal categories:

Theorem 11 From a 2-monoidal structure $\mathcal{C}$ one can construct braidings on $(\mathcal{C},(\mathbb{D})$ and $(\mathcal{C}, \ominus)$. The identity functor may be viewed as a braided monoidal functor $e:(\mathcal{C}, \oplus) \rightarrow(\mathcal{C}, \ominus)$ with unit constraint $e_{I}=\mathrm{id}$ and structure maps

$$
e_{A, B}^{\oplus, \ominus}: A \oplus B \stackrel{\rho^{\ominus} \oplus \lambda^{\ominus}}{\longleftarrow}(A \ominus I) \oplus(I \ominus B) \cong(A \oplus I) \ominus(I \oplus B) \stackrel{\rho^{\oplus} \ominus \lambda \oplus}{\longrightarrow} A \ominus B .
$$

(here $\lambda^{\oplus}, \rho^{\oplus}$ are the unit constraints on $(\mathcal{C}, \Phi)$ and similarly for $\ominus$ ).

The double loop space $\Omega^{2} A$ of a topological abelian group $A$ (base-point 0 ) is again an abelian group. Example 4 and Example 6 (i) give two different ways of viewing the fundamental groupoid $\Pi_{1} \Omega^{2} A$ as a 2-monoidal category.

Lemma 12 For every topological abelian group A, the identity functor may be endowed canonically with the structure of a 2-monoidal functor:

$$
\left(\Pi_{1} \Omega^{2} A, \oplus, \ominus, \text { const }_{0}, \mathrm{id}\right) \longrightarrow\left(\Pi_{1} \Omega^{2} A,+,+, 0, \text { id }\right)
$$

Proof Since the operations $(1)$ and + are mutually distributive, $\left(\Pi_{1} \Omega^{2} A, \Phi,+\right.$, id $)$ defines a 2-monoidal category and Theorem 11 gives a canonical monoidal structure $e_{f, g}^{\Phi,+}$ on the identity functor. Similarly, we get a monoidal structure $e_{f, g}^{\ominus,+}$. It remains to show the commutativity of (6). Suppose $\gamma, \phi:[0,1] \rightarrow[0,1]^{2}$ satisfy $\gamma<\phi$ component-wise. For $f \in \Pi_{1} \Omega^{2} A$ define a homotopy that places $f$ into the rectangles bounded by $\gamma$ and $\phi$. Viewing $f$ as a map $[0,1]^{2} \rightarrow A$ taking the boundary to zero and extended to the plane by zero, we may write

$$
\{\gamma, \phi\}_{f}(t, x, y)=f\left(\frac{x-\gamma_{1}(t)}{\phi_{1}(t)-\gamma_{1}(t)}, \frac{y-\gamma_{2}(t)}{\phi_{2}(t)-\gamma_{2}(t)}\right) .
$$

A path homotopy $\gamma^{s}<\phi^{s}$ (parameter $s$ ) gives a homotopy $\left\{\gamma^{s}, \phi^{s}\right\}_{f}$ of homotopies. For paths $u, v:[0,1] \rightarrow X$ with $u(1)=v(0)$ let $u \star v$ denote ' $u$ followed by $v$ '. Write $\alpha(t)=(1+t) / 2, \beta(t)=(1-t) / 2$ and $c(t)=c$ for fixed $c \in[0,1]$. Equation (8) defines $e_{f, g}^{\oplus,+}$ as $\{(0,0),(\alpha, 1)\}_{f}+\{(\beta, 0),(1,1)\}_{g}$. Similarly, $e_{f, g}^{\ominus,+}=$ 
$\{(0,0),(1, \alpha)\}_{f}+\{(0, \beta),(1,1)\}_{g}$. Performing the composition,

$$
\begin{aligned}
& \left(e_{f, g}^{\oplus,+}+e_{h, j}^{\oplus,+}\right) e_{f \ominus g, h \ominus j}^{\oplus,+} \\
& =\{(0,0),(\alpha, 1 / 2) \star(1, \alpha)\}_{f}+\{(0,1 / 2) \star(0, \beta),(\alpha, 1) \star(1,1)\}_{g} \\
& \quad+\{(\beta, 0) \star(0,0),(1,1 / 2) \star(1, \alpha)\}_{h}+\{(\beta, 1 / 2) \star(0, \beta),(1,1)\}_{j}, \\
& \left(e_{f, h}^{\Phi,+}+e_{g, j}^{\oplus,+}\right) e_{f \oplus h, g \oplus j}^{\ominus,+} \\
& =\{(0,0),(1 / 2, \alpha) \star(\alpha, 1)\}_{f}+\{(0, \beta) \star(0,0),(1 / 2,1) \star(\alpha, 1)\}_{g} \\
& \quad+\{(1 / 2,0) \star(\beta, 0),(1, \alpha) \star(1,1)\}_{h}+\{(1 / 2, \beta) \star(\beta, 0),(1,1)\}_{j} .
\end{aligned}
$$

These are homotopic, since any two paths in $[0,1]^{2}$ are homotopic by a linear homotopy, so $\{\gamma, \phi\}_{f} \simeq\left\{\gamma^{\prime}, \phi^{\prime}\right\}_{f}$ for any $\gamma<\phi, \gamma^{\prime}<\phi^{\prime}$ and any $f$.

\section{The cocycle spectrum of a space}

In this section, we shall construct an auxiliary object which mediates between the algebraic and homotopical point of view. We assume familiarity with simplicial sets (see [3]). Recall that the Moore complex $C(K)_{*}$ of a pointed simplicial set $K$ has the group $\mathbb{Z} K_{n} / \mathbb{Z}$ pt as $n$-chains. We adopt the standard notation $C(X)=C(\operatorname{sing} X)$ for $X \in \mathcal{T}$ op* . Let $L_{+}$denote $L$ with a disjoint base-point.

Definition 13 The $n$-th space of the cocycle spectrum is the simplicial vector space of chain maps $\left(V[-n]_{*}=V^{n-*}\right.$ for $* \geq 0$ with zero differential is viewed as an object of the category $\mathrm{Ch}_{\geq 0}$ of non-negative chain complexes):

$$
Z^{n}\left(K \wedge \Delta_{+}^{\bullet}\right)=\mathcal{C} h_{\geq 0}\left(C\left(K \wedge \Delta_{+}^{\bullet}\right)_{*}, V[-n]_{*}\right)=\prod_{i+j=n} Z^{i}\left(K \wedge \Delta_{+}^{\bullet} ; V^{j}\right)
$$

Being fixed, we omit $V$ from the notation on the left of (10).

The spaces $Z^{n}\left(K \wedge \Delta_{+}^{\bullet}\right)$ are the mapping spaces $\operatorname{Map}_{\mathrm{Ch}}(C(K), V[-n])$ in the $\infty$-category of non-negative chain complexes [5, Section 13], so the cocycle spectrum may be regarded as a function spectrum construction. Weakly equivalent spaces were introduced in [4], but we will see below that it is crucial to work with (10).

Recall the Alexander-Whitney and Eilenberg-Zilber chain maps

$$
E Z: C(K) \otimes C(L) \rightarrow C(K \wedge L), \quad A W: C(K \wedge L) \rightarrow C(K) \otimes C(L) .
$$

The slant product of a cochain $u$ with a chain $e$ is the cochain $u / e$ defined by $(u / e)(d)=u(E Z(d \otimes e))$. Since $E Z$ is a chain map, we get a Stokes formula

$$
(\delta u / e)=\delta(u / e)-(-1)^{|u|+|e|} u / \partial e .
$$

Let $\left[\Delta_{+}^{i}\right] \in C_{i}\left(\Delta_{+}^{i}\right)$ and $\left[S^{1}\right] \in C_{1}\left(S^{1}\right)$ denote the canonical chains $\left(S^{1}=\Delta^{1} / \partial \Delta^{1}\right)$. 
We take from [4, Definition 13] the isomorphism 'slant product along the $i$-chain $\left[\Delta_{+}^{i}\right]$

$$
\pi_{i}\left(Z^{n}\left(K \wedge \Delta_{+}^{\bullet}\right), 0\right) \cong H^{n-i}(K ; V), \quad f \in Z^{n}\left(K \wedge \Delta_{+}^{i}\right) \mapsto f /\left[\Delta_{+}^{i}\right] .
$$

(this fact is also proven in [9, Lemma 5.7]).

Lemma 14 There is a canonical isomorphism of simplicial sets

$$
\Omega Z^{n}\left(K \wedge \Delta_{+}^{\bullet}\right) \cong Z^{n}\left(K \wedge \Delta_{+}^{\bullet} \wedge S^{1}\right)
$$

Proof The usual subdivision of the prism $h_{i}: \Delta^{k+1} \rightarrow \Delta^{k} \times \Delta^{1}$ for $i=0, \ldots, k[3$, pp. 17-18] leads to a coequalizer diagram in pointed simplicial sets $\mathcal{S e t}_{\Delta}^{*}$ :

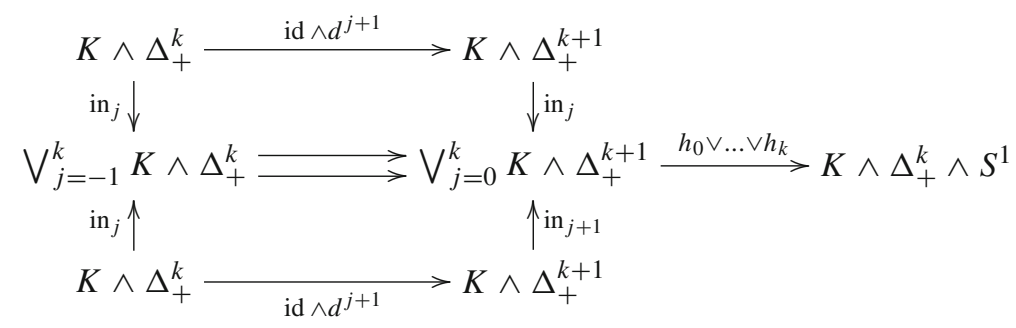

(letting in $\operatorname{in}_{l}$ be the constant base-point maps if $l=-1, k+1$ ). The reduced Moore complex $C: \mathcal{S e t}_{\Delta}^{*} \rightarrow \mathcal{C h}_{\geq 0}$ is a left-adjoint and therefore preserves colimits. Hence a $k$-simplex $f \in Z^{n}\left(K \wedge \Delta_{+}^{k} \wedge S^{1}\right)$ is a chain map defined on the coequalizer of

$$
\bigoplus_{j=-1}^{k} C\left(K \wedge \Delta_{+}^{k}\right) \longrightarrow \bigoplus_{j=0}^{k} C\left(K \wedge \Delta_{+}^{k+1}\right) .
$$

This amounts to a sequence of maps $f_{i} \in Z^{n}\left(K \wedge \Delta_{+}^{k+1}\right)$ which are compatible exactly so as to represent a $k$-simplex of the loop space $\Omega Z^{n}\left(K \wedge \Delta_{+}^{\bullet}\right)$ (a $k$-simplex of a simplicial loop space $\Omega L$ may be described as a sequence of $(k+1)$-simplices $f_{0}, \ldots, f_{k}$ with $d_{i} f_{i}=d_{i} f_{i-1}$ and $\left.d_{0} f_{0}=d_{k+1} f_{k}=*\right)$.

Definition 15 Letting 'incl' be given by the canonical 1-chain $\left[S^{1}\right]$, consider

$$
C\left(K \wedge \Delta_{+}^{\bullet}\right) \otimes \mathbb{Z}[1] \stackrel{\text { id } \otimes \text { incl }}{\longrightarrow} C\left(K \wedge \Delta_{+}^{\bullet}\right) \otimes C\left(S^{1}\right) \stackrel{E Z}{\longrightarrow} C\left(K \wedge \Delta_{+}^{\bullet} \wedge S^{1}\right) .
$$

Combining that $-\otimes \mathbb{Z}[1]$ is the shift $[-1]$ with Lemma 14, pullback along (14) gives the costructure maps ('co' because they map away from the loop space)

$$
\psi: \Omega Z^{n}\left(K \wedge \Delta_{+}^{\bullet}\right) \cong Z^{n}\left(K \wedge \Delta_{+}^{\bullet} \wedge S^{1}\right) \rightarrow Z^{n-1}\left(K \wedge \Delta_{+}^{\bullet}\right) .
$$

Proposition 16 The costructure maps $\psi$ are natural weak equivalences. 
Proof This follow from the standard fact that the suspension may be expressed as the slant product along $S^{1}$ : we show that we have commutative diagrams

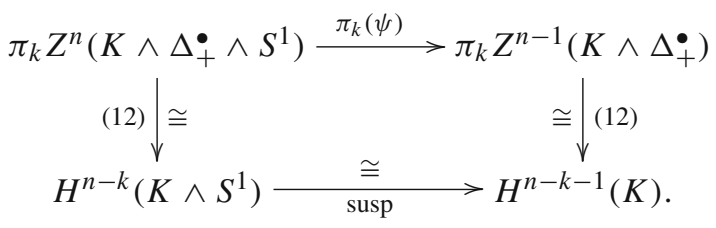

Explicitly, for $f \in \pi_{k} Z^{n}\left(K \wedge \Delta_{+}^{\bullet} \wedge S^{1}\right)$ we need to compare the two assignments on chains $\sigma \in C_{n-k-1}(K)$ given by

$$
f \circ E Z\left(\Delta^{k} \otimes E Z\left(\sigma \otimes S^{1}\right)\right), \quad f \circ E Z\left(E Z\left(\Delta^{k} \otimes \sigma\right) \otimes S^{1}\right) .
$$

Since $E Z$ is coassociative up to chain homotopy, we have a homomorphism $h$ so that the difference is (using that $f$ is a chain map and $V_{*}$ has zero differential)

$$
f \circ(\partial h \sigma+h \partial \sigma)=\partial f(h \sigma)+f h \partial \sigma=0+\delta(f h) \sigma
$$

Therefore, both elements in (16) represent the same cohomology class.

Definition 17 By the costructure maps on $\operatorname{sing}\left(E_{n}^{X}\right)$ we mean the isomorphisms

$$
\Omega \operatorname{sing}\left(E_{n+1}^{X}\right) \cong \operatorname{sing}\left(\Omega E_{n+1}^{X}\right) \stackrel{\operatorname{sing}\left(\varepsilon_{n}^{\mathrm{adj}}\right)_{*}^{-1}}{\longrightarrow} \operatorname{sing}\left(E_{n}^{X}\right) .
$$

\section{The 2-monoidal Chern-Dold transformation}

Our construction of (2) will factor into three 2-monoidal functors

$$
\operatorname{ch}_{X}: \mathcal{M a p}\left(X, E_{n}\right) \stackrel{\alpha}{\rightarrow} \mathscr{Z}_{\oplus, \ominus}^{n}(X) \stackrel{\beta}{\rightarrow} \mathscr{Z}_{+}^{n}(X) \stackrel{\gamma}{\rightarrow} \mathscr{Z}^{n}(X) .
$$

We begin by explaining the new categories in (18). By Example 6 (i), addition gives a strict 2-monoidal structure on the fundamental groupoid $\Pi_{1} Z^{n}\left(\operatorname{sing} X \wedge \Delta_{+}^{\bullet}\right)$ that we denote by $\mathscr{Z}_{+}^{n}(X)$. Hence the objects of $\mathscr{Z}_{+}^{n}(X)$ are singular cocycles $Z^{n}(X)$ while the morphisms $h: d_{1} h \rightarrow d_{0} h$ are cocycles $h \in Z^{n}\left(\operatorname{sing} X \wedge \Delta_{+}^{1}\right)$. Another way to get a 2-monoidal structure on the same category is to note that the costructure map $\psi$ induces equivalences of categories

$$
\Pi_{1} \Omega^{2} Z^{n+2}\left(\operatorname{sing} X \wedge \Delta_{+}^{\bullet}\right) \stackrel{\sim}{\rightarrow} \Pi_{1} Z^{n}\left(\operatorname{sing} X \wedge \Delta_{+}^{\bullet}\right) .
$$

The left-hand side has a natural 2-monoidal structure by Example 10. According to Proposition 9, we may choose natural 2-monoidal structures $\mathscr{Z}_{\oplus, \ominus}^{n}(X)$ on the righthand categories, making (19) a natural 2-monoidal equivalence. 


\subsection{Fundamental cocycles}

Recall that fundamental cocycles are a family of singular cocycles $\iota_{n} \in Z^{n}\left(E_{n} ; V\right)$ implementing the Chern-Dold character via

$$
\operatorname{ch}(f)=f^{*}\left[\iota_{n}\right], \quad \forall f \in E^{n}(X)=\left[X, E_{n}\right] .
$$

By [4, 4.8], there is a choice satisfying $\varepsilon_{n}^{*} \iota_{n+1} /\left[S^{1}\right]=\iota_{n}$, where $\varepsilon_{n}: E_{n} \wedge S^{1} \rightarrow E_{n+1}$ are the structure maps (a more detailed proof of this assertion may be found in [9, Section 3.1.2]). Stated differently, we have chain maps

$$
\iota_{n}: C\left(E_{n}\right)=C\left(\operatorname{sing} E_{n}\right) \rightarrow V[-n]
$$

fitting into commutative diagrams

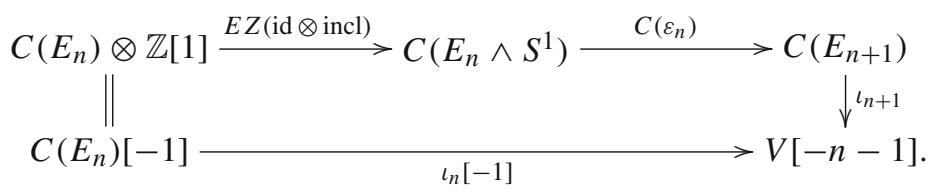

Definition 18 We define simplicial maps $A_{n}: \operatorname{sing}\left(E_{n}^{X}\right) \rightarrow Z^{n}\left(\operatorname{sing} X \wedge \Delta_{+}^{\bullet}\right)$ by

$$
A_{n}(f): C\left(\operatorname{sing} X \wedge \Delta_{+}^{k}\right) \stackrel{C\left(f^{\mathrm{adj}}\right)}{\longrightarrow} C\left(\operatorname{sing} E_{n}\right) \stackrel{\iota_{n}}{\longrightarrow} V[-n]
$$

where, for a $k$-simplex $f: X \wedge\left|\Delta_{+}^{k}\right| \rightarrow E_{n}$ of $\operatorname{sing}\left(E_{n}^{X}\right)$, we use the unit to write

$$
f^{\text {adj }}: \operatorname{sing} X \wedge \Delta_{+}^{k} \rightarrow \operatorname{sing} X \wedge \operatorname{sing}\left|\Delta_{+}^{k}\right|=\operatorname{sing}\left(X \wedge\left|\Delta_{+}^{k}\right|\right) \stackrel{\operatorname{sing} f}{\longrightarrow} \operatorname{sing} E_{n} .
$$

Lemma 19 The maps $A_{n}$ commute with the costructure maps:

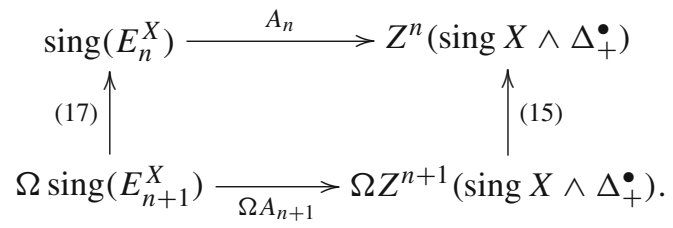

Proof For $f: X \wedge\left|\Delta_{+}^{\bullet}\right| \wedge S^{1} \rightarrow E_{n+1}$ let $g: X \wedge\left|\Delta_{+}^{\bullet}\right| \rightarrow E_{n}$ be the map $\left(\varepsilon_{n}^{\text {adj }}\right)^{-1} f$ with $\varepsilon_{n} \circ\left(g \wedge 1_{S^{1}}\right)=f$. If we write $K=\operatorname{sing} X$, the counit gives a simplicial map

$$
\varphi(f): K \wedge \Delta_{+}^{\bullet} \wedge S^{1} \rightarrow \operatorname{sing}\left(X \wedge\left|\Delta_{+}^{\bullet}\right| \wedge S^{1}\right) \rightarrow \operatorname{sing} E_{n+1} .
$$


Unwinding the definitions of (15), (17), and $A$, we see that we need to compare

$$
\begin{gathered}
C\left(K \wedge \Delta_{+}^{\bullet}\right) \otimes \mathbb{Z}[1] \stackrel{1 \otimes \text { incl }}{\longrightarrow} C\left(K \wedge \Delta_{+}^{\bullet}\right) \otimes C\left(S^{1}\right) \stackrel{E Z}{\longrightarrow} C\left(K \wedge \Delta_{+}^{\bullet} \wedge S^{1}\right) \\
\stackrel{\varphi(f)_{*}}{\longrightarrow} C\left(E_{n+1}\right) \stackrel{\iota_{n+1}}{\longrightarrow} V[-n-1]
\end{gathered}
$$

with the shift by one of

$$
C\left(K \wedge \Delta_{+}^{\bullet}\right) \stackrel{\varphi(g)_{*}}{\longrightarrow} C\left(E_{n}\right) \stackrel{\iota_{n}}{\longrightarrow} V[-n] .
$$

But these maps appear as the outer maps in the diagram

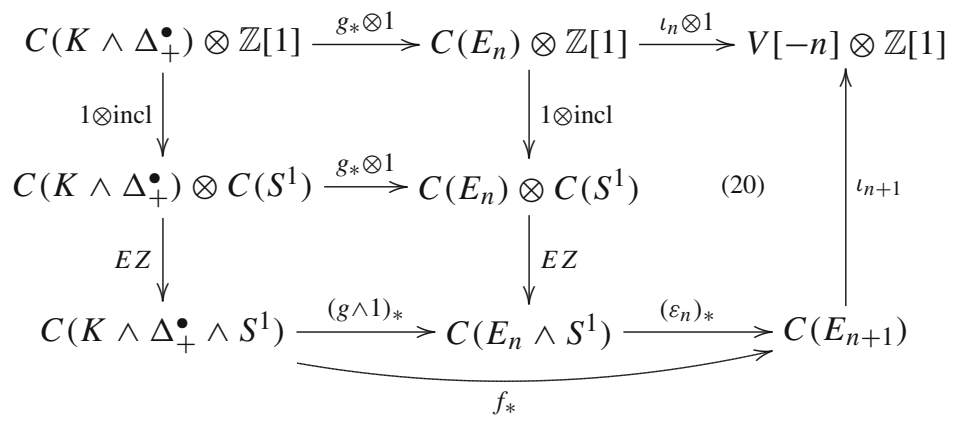

which commutes by naturality of $E Z$ and the compatibility (20).

\subsection{Proof of Theorem 1}

The proof is divided into three steps:

Lemma 20 The maps $A_{n}$ induce a natural family of 2-monoidal functors

$$
\Pi_{1} A_{n}=\alpha_{X}: \mathcal{M a p}\left(X, E_{n}\right) \rightarrow \mathscr{Z}_{\oplus, \ominus}^{n}(X)
$$

Proof Lemma 19 asserts that the diagram of ordinary categories underlying

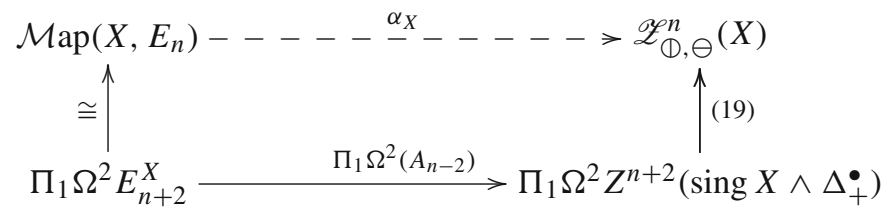

commutes. On the bottom is the 2-monoidal functor $\Pi_{1} \Omega^{2}$ from Example 10 and the vertical functors are 2-monoidal by definition of the 2-monoidal structure on the categories upstairs. Proposition 8 states that there is a unique way to put a 2-monoidal structure $\alpha_{X}$ on $\Pi_{1} A$ so as make this diagram commute in $2 \mathcal{M}$ on. Uniqueness allows 
us to conclude the naturality (in $X$ ) of this structure from the naturality of the 2monoidal structure on the other arrows.

Lemma 21 The identity functor $\Pi_{1} Z^{n}\left(\operatorname{sing} X \wedge \Delta_{+}^{\bullet}\right)$ has a unique (hence natural) 2-monoidal structure $\beta_{X}$ making the diagram

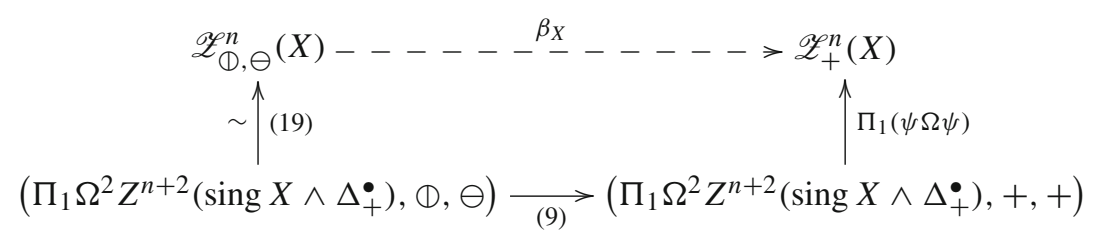

commute as a diagram of 2-monoidal categories and functors.

The right vertical map is 2-monoidal since $\psi$ is linear. The proof of Lemma 21 is now immediate from Proposition 8.

Both categories $\mathscr{Z}_{+}^{n}(X), \mathscr{Z}^{n}(X)$ have the same objects $Z^{n}(X)$ and we let $\gamma_{X}$ be the identity on objects. To a morphism $f \in Z^{n}\left(\operatorname{sing} X \wedge \Delta_{+}^{1}\right)$ in $\mathscr{Z}_{+}^{n}(X)$ from $d_{1} f$ to $d_{0} f$ we assign the class of the cochain $f /\left[\Delta^{1}\right] \in C^{n-1}(X ; V) / \operatorname{im}(\delta)$.

Lemma $22 \gamma_{X}: \mathscr{Z}_{+}^{n}(X) \rightarrow \mathscr{Z}^{n}(X)$ is a well-defined strict 2-monoidal functor.

Proof In the fundamental groupoid, a composition $f \circ g=h$ is 'witnessed' by a 2-simplex $\sigma \in Z^{n}\left(\operatorname{sing} X \wedge \Delta_{+}^{2}\right)$, meaning $\partial \sigma=g-h+f$. Hence (11) implies that

$$
(-1)^{n} \delta\left(\sigma /\left[\Delta^{2}\right]\right)=\sigma / \partial\left[\Delta^{2}\right]=g /\left[\Delta^{1}\right]-h /\left[\Delta^{1}\right]+f /\left[\Delta^{1}\right]
$$

is a coboundary, which proves that $\gamma_{X}$ is a functor. To show that $\gamma_{X}$ is well-defined, let $\sigma$ be a homotopy from $d_{0} \sigma=f$ to $d_{1} \sigma=f^{\prime}$ with $d_{2} \sigma=0$. Then $(-1)^{n} \delta\left(\sigma /\left[\Delta^{2}\right]\right)=$ $f /\left[\Delta^{1}\right]-f^{\prime} /\left[\Delta^{1}\right]+0$ exhibits the required coboundary. Since taking slant products is linear, $\gamma_{X}$ is strict 2-monoidal.

Combining Lemmas 20, 21, 22, we define $\operatorname{ch}_{X}$ to be the composite 2-monoidal functor $\gamma_{X} \beta_{X} \alpha_{X}$. Explicitly, $\mathrm{ch}_{X}$ is given on objects and morphisms as follows:

$\operatorname{ch}_{X}: \mathcal{M a p}\left(X, E_{n}\right) \rightarrow \mathscr{Z}^{n}(X), \quad \begin{cases}\text { objects } f: & \operatorname{ch}(f)=f^{*} \iota_{n}, \\ \text { morphisms } H: f \simeq g: & \operatorname{ch}(H)=H^{*} \iota_{n} /\left[\Delta^{1}\right] .\end{cases}$

In particular, $\operatorname{ch}_{X}$ recovers (1) on isomorphism classes of objects. $\alpha_{X}, \beta_{X}, \gamma_{X}$ are natural in $X$, so this holds for $\mathrm{ch}_{X}$, too. This completes the proof.

\section{Application to differential cohomology}

We begin by unravelling parts of Theorem 1 into more elementary form. As shown in [6, Section 5], there is an equivalence $2 \mathcal{M}$ on $\rightarrow \mathcal{M}$ onC at $_{\text {braid }}$ to braided monoidal categories. Hence we regard $\mathcal{M} \operatorname{ap}\left(X, E_{n}\right)$ as having just a single monoidal structure 
(1) and a natural braid (given by Theorem 11) and the functors $\operatorname{ch}_{X}$ from Theorem 1 as having a natural braided monoidal structure $s$.

Fix the standard homotopies showing that $\pi_{0} \Omega^{2} E_{n+2}$ is an abelian group (so the associator $a=a_{\mathrm{pr}_{1}, \mathrm{pr}_{2}, \mathrm{pr}_{3}}$ in $\mathcal{M a p}\left(E_{n}^{\times 3}, E_{n}\right)$, braid $s=s_{\mathrm{pr}_{1}, \mathrm{pr}_{2}}$ in $\mathcal{M a p}\left(E_{n}^{\times 2}, E_{n}\right)$, and unit constraint $r=r_{\text {id }}$ in $\left.\mathcal{M a p}\left(E_{n}, E_{n}\right)\right)$ :

$$
\begin{aligned}
a: E_{n}^{\times 3} \times I \rightarrow E_{n}, & & \alpha_{n} \circ\left(\alpha_{n} \times \mathrm{id}\right) \simeq \alpha_{n} \circ\left(\mathrm{id} \times \alpha_{n}\right), \\
s: E_{n}^{\times 2} \times I \rightarrow E_{n}, & & \alpha_{n} \circ \text { flip } \simeq \alpha_{n}, \\
r: E_{n} \times I \rightarrow E_{n}, & & \alpha_{n} \circ(\mathrm{id}, \text { const }) \simeq \mathrm{id} .
\end{aligned}
$$

The monoidal structure $(1)$ was induced by horizontal concatenation of loops:

$$
\alpha_{n}: E_{n} \times E_{n} \approx \Omega^{2} E_{n+2} \times \Omega^{2} E_{n+2} \rightarrow \Omega^{2} E_{n+2} \approx E_{n}
$$

Then (either by direct inspection or using the naturality in $X$ ), the associativity and unit constraints $a, r$ as well as the braid $s$ on the categories $\mathcal{M a p}\left(X, E_{n}\right)$ are given by post-composition with the above homotopies.

Theorem 23 There exist reduced cochains $A_{n} \in C^{n-1}\left(E_{n} \times E_{n} ; V\right)$ satisfying

$$
\delta A_{n}=\operatorname{pr}_{1}^{*} \iota_{n}+\operatorname{pr}_{2}^{*} \iota_{n}-\alpha_{n}^{*} \iota_{n}
$$

and coherent in the sense that ('三' means 'up to coboundary')

$$
\begin{aligned}
\operatorname{pr}_{12}^{*} A_{n}+\left(\alpha_{n} \times 1\right)^{*} A_{n} & \equiv \operatorname{pr}_{23}^{*} A_{n}+\left(1 \times \alpha_{n}\right)^{*} A_{n}+\operatorname{ch}(a), & & \text { associative } \\
\text { flip }^{*} A_{n} & \equiv A_{n}+\operatorname{ch}(s), & & \text { commutative } \\
\left(\operatorname{id}_{E_{n}}, \text { const }\right)^{*} A_{n} & \equiv \operatorname{ch}(r) . & & \text { unit }
\end{aligned}
$$

(Recall that $\operatorname{ch}(h)=h^{*} \iota_{n} /[0,1]=\int_{0}^{1} h^{*} \iota_{n}$ for morphisms/homotopies $h$ ).

Proof The data of a monoidal functor

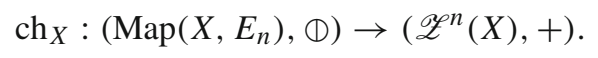

includes morphisms relating $\Phi,+$; that is, elements $\operatorname{ch}_{c, d}^{\oplus} \in C^{n-1}(X) / \operatorname{im}(\delta)$ with

$$
\delta \operatorname{ch}_{c, d}^{\oplus}=\operatorname{ch}(f \oplus g)-\operatorname{ch}(f)-\operatorname{ch}(g) .
$$

Naturality gives commutative diagrams of braided monoidal functors

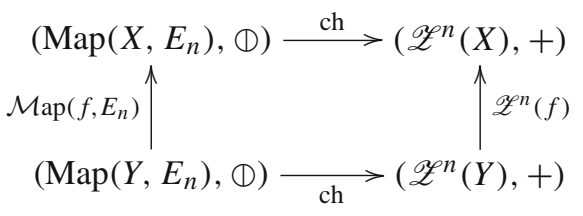


which means

$$
f^{*} \operatorname{ch}_{c, d}^{\Phi}=\operatorname{ch}_{f^{*} c, f^{*} d}^{\Phi} \quad \text { in } \quad C^{n-1}(X) / \operatorname{im}(\delta), \quad c, d: Y \rightarrow E_{n} .
$$

A braided monoidal functor has to satisfy various coherence conditions:
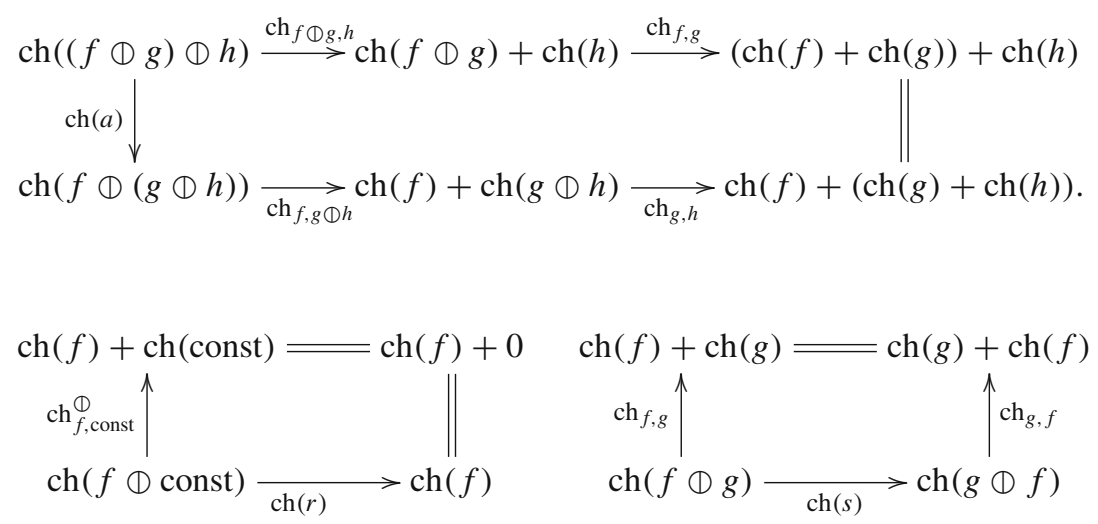

Set $c=\operatorname{pr}_{1}, d=\operatorname{pr}_{2}: E_{n} \times E_{n} \rightarrow E_{n}$ in (22) to define

$$
A_{n}=\operatorname{ch}_{\mathrm{pr}_{1}, \mathrm{pr}_{2}}^{\oplus}
$$

With this notation, the commutativity of the first coherence diagram reads

$$
\operatorname{ch}_{g, h}^{\Phi}+\operatorname{ch}_{f, g \oplus h}^{\oplus}+\operatorname{ch}(a) \equiv \operatorname{ch}_{f, g}^{\Phi}+\operatorname{ch}_{f \oplus g, h}^{\Phi} \quad \text { in } C^{n-1}(X) / \operatorname{im}(\delta) .
$$

If we set $f=\mathrm{pr}_{1}, g=\mathrm{pr}_{2}, h=\mathrm{pr}_{3}: E_{n} \times E_{n} \times E_{n} \rightarrow E_{n}$, naturality (23) asserts

$$
\begin{aligned}
& f=\left(1 \times \alpha_{n}\right)^{*} \operatorname{pr}_{1}, g \oplus h=\left(1 \times \alpha_{n}\right)^{*} \mathrm{pr}_{2} \Rightarrow \operatorname{ch}_{f, g \oplus h}^{\Phi} \equiv\left(1 \times \alpha_{n}\right)^{*} \mathrm{ch}_{\mathrm{pr}_{1}, \mathrm{pr}_{2}}^{\Phi} \text {, } \\
& g=\operatorname{pr}_{23}^{*} \operatorname{pr}_{1}, h=\operatorname{pr}_{23}^{*} \operatorname{pr}_{2} \Rightarrow \mathrm{ch}_{g, h}^{\Phi} \equiv \mathrm{pr}_{23}^{*} \mathrm{ch}_{\mathrm{pr}_{1}, \mathrm{pr}_{2}}^{\Phi}, \\
& f\left(g=\left(\alpha_{n} \times 1\right)^{*} \mathrm{pr}_{1}, h=(\alpha \times 1)^{*} \mathrm{pr}_{2} \Rightarrow \operatorname{ch}_{f \oplus g, h}^{\oplus} \equiv\left(\alpha_{n} \times 1\right)^{*} \operatorname{ch}_{\mathrm{pr}_{1}, \mathrm{pr}_{2}}^{\oplus}\right. \text {, } \\
& f=\mathrm{pr}_{12}^{*} \mathrm{pr}_{1}, g=\mathrm{pr}_{12}^{*} \mathrm{pr}_{2} \Rightarrow \mathrm{ch}_{f, g}^{\oplus} \equiv \mathrm{pr}_{12}^{*} \mathrm{ch}_{\mathrm{pr}_{1}, \mathrm{pr}_{2}}^{\oplus} \text {. }
\end{aligned}
$$

Inserting these equalities and (24) into (25) gives

$$
\operatorname{pr}_{23}^{*} A_{n}+\left(1 \times \alpha_{n}\right)^{*} A_{n}+\operatorname{ch}(a) \equiv \operatorname{pr}_{12}^{*} A_{n}+\left(\alpha_{n} \times 1\right)^{*} A_{n}
$$

Similarly, the second coherence diagram for $f=\mathrm{pr}_{1}$ asserts

$$
\operatorname{ch}(r) \equiv \operatorname{ch}_{\mathrm{pr}_{1}, \text { const }}^{\oplus} \stackrel{(23)}{\equiv}(\mathrm{id}, \text { const })^{*} \operatorname{ch}_{\mathrm{pr}_{1}, \mathrm{pr}_{2}}=(\mathrm{id}, \text { const })^{*} A_{n}
$$

The third diagram for $f=\mathrm{pr}_{1}, g=\mathrm{pr}_{2}$ says, using naturality (23) for $\mathrm{pr}_{2}=$ flip* $\mathrm{pr}_{1}, \mathrm{pr}_{1}=$ flip* $^{*} \mathrm{pr}_{2}$ : 


$$
\text { flip* }^{*}+\operatorname{ch}(s) \equiv \text { flip* }^{*} \operatorname{ch}_{f, g}+\operatorname{ch}(s) \equiv \operatorname{ch}_{g, f}+\operatorname{ch}(s) \equiv \operatorname{ch}_{f, g}=A_{n}
$$

Theorem 23 contains exactly the coherence conditions needed to prove that (3) gives an abelian group structure. The key observation is (see [9, 3.10, 3.13]):

Proposition 24 (i) Given a homotopy $C: c_{0} \simeq c_{1}$ of maps, a form $\omega \in \Omega_{\mathrm{cl}}^{n}(M ; V)$, and cochain $h \in C^{n-1}(M ; V)$ with $\delta h=\omega-c_{0}^{*} \iota_{n}$, we have an equivalence

$$
\left(c_{0}, \omega, h\right) \sim\left(c_{1}, \omega, h-\operatorname{ch}(C)\right) .
$$

(ii) For a cocycle $(c, \omega, h)$ and $g \in C^{n-2}(M ; V)$ we have $(c, \omega, h) \sim(c, \omega, h+\delta g)$.

\subsection{Proof of Theorem 2}

Applying part (i) to the homotopies $a, r, s$ above and then part (ii) to the coherence equations in Theorem 23 shows that (3) descends to an associative, unital, and commutative operation on equivalence classes.

It remains to show that we have inverses. Pick maps $v_{n}: E_{n} \rightarrow E_{n}$ representing negation in $E$-cohomology and a homotopy $h: \operatorname{id}_{n} \oplus v_{n}=\alpha_{n}\left(\operatorname{id}_{n}, v_{n}\right) \simeq$ const. For

$$
N_{n}=\operatorname{ch}(h)-\left(\mathrm{id}, v_{n}\right)^{*} A_{n}
$$

we have $\delta N_{n}=-\iota_{n}-v_{n} \iota_{n}$. Applying Proposition 24 to the homotopy $h$ then shows that $(c, \omega, h)+\left(v_{n} \circ c,-\omega,-h+c^{*} N_{n}\right)$ is equivalent to zero.

\section{Appendix}

From [7] (or see [9, Appendix A]) we recall the following well-known fact:

Theorem 25 (Doctrinal Adjunction) Suppose $(F, G, \varepsilon, \eta)$ is an adjoint equivalence in which $F: \mathcal{C} \rightarrow \mathcal{D}$ is a monoidal functor. Then there exists a unique monoidal structure on $G$ that makes $(F, G, \varepsilon, \eta)$ a monoidal adjoint equivalence.

Lemma 26 Suppose $G \circ F=H$ are functors, where $F, H$ are monoidal and $F$ is an equivalence. There exists a unique monoidal structure on $G$ so that $G \circ F=H$ as monoidal functors. (similarly, if $G$ is an equivalence, $G, H$ monoidal).

Proof If a monoidal structure on $G$ exists, we must have $H_{C_{1}, C_{2}}=G\left(F_{C_{1}, C_{2}}\right) \circ$ $G_{F C_{1}, F C_{2}}$ and $H_{1}=G\left(F_{1}\right) \circ G_{1}$. Hence $G_{E_{1}, E_{2}}$ is determined on the image of $F$. For general $E_{1}, E_{2}$ pick isomorphisms $\varphi_{i}: E_{i} \rightarrow F C_{i}$. Naturality gives $G_{F C_{1}, F C_{2}} \circ\left(G \varphi_{1} \otimes G \varphi_{2}\right)=G\left(\varphi_{1} \otimes \varphi_{2}\right) \circ G_{E_{1}, E_{2}}$, so $G_{E_{1}, E_{2}}$ is uniquely determined. To prove existence, place $F$ in an adjoint equivalence $(F, R, \varepsilon, \eta)$ which, by doctrinal adjunction, may viewed as a monoidal adjunction. We have a natural isomorphism $G \varepsilon: H R=G F R \stackrel{\cong}{\longrightarrow} G$. There is a unique monoidal structure on $G$ making $G \varepsilon$ a monoidal transformation (monoidal structures on functors may be uniquely 
transported along natural isomorphisms). The composition of a monoidal transformation with a monoidal functor is again monoidal. Therefore $H \eta: H \rightarrow H R F$, $G \varepsilon F: H R F \rightarrow G F$ are monoidal transformations which compose to the identity, by the zig-zag identities for $(F, R, \varepsilon, \eta)$. But this just means $H=G F$ as monoidal functors.

\section{Proof of Proposition 8}

We prove only 1 . since 2 . follows by a dual argument. Placing $F$ in an adjoint equivalence $(F, R, \varepsilon, \eta)$, Lemma 26 gives us two monoidal structures on $G$ satisfying $G^{\oplus} \circ F^{\oplus}=H^{\oplus}$ and $G^{\ominus} \circ F^{\ominus}=H^{\ominus}$. Since $H, F$ preserve $\zeta$, we have [omitting the vertical maps in (6) from the notation]

$$
\zeta_{H A_{1}, H A_{2}, H A_{3}, H A_{4}}^{\mathcal{E}}=H \zeta_{A_{1}, A_{2}, A_{3}, A_{4}}^{\mathcal{C}}=G F\left(\zeta_{A_{1}, A_{2}, A_{3}, A_{4}}^{\mathcal{C}}\right)=G\left(\zeta_{F A_{1}, F A_{2}, F A_{3}, F A_{4}}^{\mathcal{D}}\right) .
$$

Hence $G$ preserves the interchange on the image of $F$. In general, we may pick isomorphisms $X_{i} \cong F A_{i}$ ( $F$ is essentially surjective). Naturality of the interchange then implies that $G$ preserves $\zeta_{X_{1}, X_{2}, X_{3}, X_{4}}^{\mathcal{D}}$. Hence $G$ is 2-monoidal.

We will use the following well-known fact (see [9, Appendix A]):

Proposition 27 Let $(F, G, \varepsilon, \eta)$ be an adjoint equivalence from $\mathcal{C}$ to $\mathcal{D}$ and assume that $\mathcal{D}$ is a monoidal category. Then there exists a monoidal structure on $\mathcal{C}$ making $(F, G, \varepsilon, \eta)$ a monoidal adjoint equivalence.

(for example, one can set $\left.C_{1} \otimes C_{2}=G\left(F C_{1} \otimes F C_{2}\right), I_{\mathcal{C}}=G I_{\mathcal{D}}\right)$.

Applied to both monoidal structures of a 2-monoidal category $\mathcal{D}$, we obtain two monoidal adjunctions $\left(F^{\oplus}, G^{\oplus}, \varepsilon, \eta\right)$ and $\left(F^{\ominus}, G^{\ominus}, \varepsilon, \eta\right)$. As (6) is a diagram of isomorphisms and $F$ is bijective on Hom-sets, there is a unique interchange $\zeta^{\mathcal{C}}$ making $F$ a 2-monoidal functor. The verification of the diagrams (5) expressing the compatibility of $\zeta^{\mathcal{C}}$ with associativity and unit constraints can, $F$ being faithful, be reduced to the corresponding properties of $\zeta^{\mathcal{D}}$.

\section{Proof of Proposition 9}

Placing the equivalences $F_{i}: \mathcal{C}_{i} \rightarrow \mathcal{D}_{i}$ into an adjoint equivalence $\left(F_{i}, G_{i}, \varepsilon_{i}, \eta_{i}\right)[8$, Proposition 1.1.2], the preceding remark gives 2-monoidal structures $\hat{\mathcal{C}}_{i}$ on $\mathcal{C}_{i}$ and $\hat{F}_{i}$ on every $F_{i}$. It remains to define 2-monoidal structures on every $\mathcal{C}(f): \mathcal{C}_{i} \rightarrow \mathcal{C}_{j}$ for morphisms $f: i \rightarrow j$ in $\mathcal{I}$. The naturality of $\hat{F}_{i}$ means that

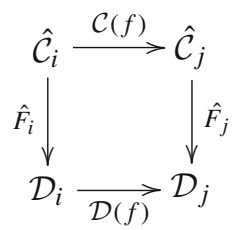

should commute as a diagram in $2 \mathcal{M}$ on. Proposition 8 implies that there is a unique such 2-monoidal structure on $\mathcal{C}(f)$. Uniqueness implies functoriality. 


\section{References}

1. Aguiar, M., Mahajan, S.: Monoidal Functors, Species and Hopf Algebras, Volume 29 of CRM Monograph Series. American Mathematical Society, Providence, RI, 2010. With forewords by Kenneth Brown and Stephen Chase and André Joyal (2010)

2. Dold, A.: Algebraic Topology-A Students's Guide, Relations between ordinary and extraordinary homology. London Mathematical Society Lecture Note Series, No. 4, pp. 166-177. Cambridge University Press, London (1972)

3. Goerss, P.G., Jardine, J.F.: Simplicial Homotopy Theory. Modern Birkhäuser Classics. Birkhäuser Verlag, Basel (2009). Reprint of the 1999 edition [MR1711612]

4. Hopkins, M.J., Singer, I.M.: Quadratic functions in geometry, topology, and M-theory. J. Differ. Geom. 70(3), 329-452 (2005)

5. Jacob, L.: Stable Infinity Categories (2007). arXiv preprint math.CT/0608228

6. Joyal, A., Street, R.: Braided tensor categories. Adv. Math. 102(1), 20-78 (1993)

7. Kelly, G.M.: Doctrinal adjunction. In: Category Seminar (Proc. Sem., Sydney, 1972/1973). Lecture Notes in Math., vol. 420, pp. 257-280. Springer, Berlin (1974)

8. Leinster, T.: Higher Operads, Higher Categories. London Mathematical Society Lecture Note Series, vol. 298. Cambridge University Press, Cambridge (2004)

9. Markus, U. Algebraic Structure and Integration in Generalized Differential Cohomology. PhD thesis, Georg-August Universität Göttingen (2013) 